\title{
Considerations About Antenna Pattern Measurements of 2-D Aperture Synthesis Radiometers
}

\author{
Adriano Camps, Senior Member, Niels Skou, Fellow, Francesc Torres, Member, Ignasi Corbella, Member, \\ Núria Duffo, Life Senior Member, and Mercè Vall-llossera, Member
}

\begin{abstract}
Accurate measurement of the antenna voltage patterns of large-aperture synthesis radiometers is critical in order to achieve good radiometric accuracy, and a very time consuming and expensive task. Measurement requirements and a tradeoff study relating radiometric accuracy degradation and number of elements to be measured are presented.
\end{abstract}

Index Terms-Antenna pattern, interferometry, measurements, radiometry.

\section{INTRODUCTION}

$\mathbf{T}$ HE Microwave Imaging Radiometer by Aperture Synthesis (MIRAS) instrument aboard the Surface Moisture and Ocean Salinity (SMOS) mission of the European Space Agency (ESA) (http://www.esa.int/export/esaLP/smos.html) is a two-dimensional aperture synthesis radiometer for Earth observation (Fig. 1). In such an instrument, the observables are the so-called samples of the visibility function $\left(V_{12}^{p q}\right)$, measured by every pair of elements in the array [1]. The equation that relates the brightness temperature and the samples of the visibility function is

$$
\begin{aligned}
V_{12}^{p q}\left(u_{12}, v_{12}\right) \triangleq & \frac{1}{k_{B} \sqrt{B_{1} B_{2}} \sqrt{G_{1} G_{2}}} \cdot \frac{1}{2}\left\langle b_{1}^{p}(t) b_{2}^{q *}(t)\right\rangle \\
= & \frac{1}{\sqrt{\Omega_{1} \Omega_{2}}} \iint_{\xi_{2}+\eta^{2} \leq 1} \frac{T_{B}^{p q}(\xi, \eta)-T_{\mathrm{rec}} \delta_{p q}}{\sqrt{1-\xi^{2}-\eta^{2}}} \\
& \times F_{n 1}^{p}(\xi, \eta) F_{n 2}^{q *}(\xi, \eta) \tilde{r}_{12}\left(-\frac{u_{12} \xi+v_{12} \eta}{f_{0}}\right) \\
& \times \exp \left(-j 2 \pi\left(u_{12} \xi+v_{12} \eta\right)\right) d \xi d \eta
\end{aligned}
$$

where $k_{B}$ is the Boltzmann's constant, $B_{1,2}$ and $G_{1,2}$ are the receivers noise bandwidth and power gain, $\Omega_{1,2}$ is the antennas' solid angle, $T_{B}(\xi, \eta)$ is the brightness temperature, $T_{\mathrm{rec}}$ is the receivers' physical temperature, $\delta_{p q}=1$ if $p=q$, and 0 if not, $F_{n 1,2}^{p, q}(\xi, \eta)$ are the normalized antenna copolar voltage patterns at $p$ and $q$ polarizations, $\tilde{r}_{12}\left(-\left(u_{12} \xi+v_{12} \eta\right) / f_{0}\right)$ is the fringe-wash function, $f_{0}$ is the center frequency $\left(\lambda_{0}=c / f_{0}\right)$, $\left(u_{12}, v_{12}\right) \hat{=}\left(x_{2}-x_{1}, y_{2}-y_{1}\right) / \lambda_{0}$ is the spatial frequency,

Manuscript received May 10, 2004; revised August 24, 2004. This work was supported in part by the Spanish Government under Grants MCYT and EU FEDER TIC2002-04451-C02-01 and in part by the EADS-CASA Espacio in the frame of SMOS PLM Phase C/D activities under Grant DE03/C-181/PE1.

A. Camps, F. Torres, I. Corbella, N. Duffo, and M. Vall-1lossera are with the Department of Signal Theory and Communications, Universitat Politècnica de Catalunya, 08034 Barcelona, Spain (e-mail: camps@tsc.upc.es).

N. Skou is with the Oersted-DTU/Electromagnetic Systems Section, Technical University of Denmark, DK 2800 Lyngby, Denmark.

Digital Object Identifier 10.1109/LGRS.2006.870336

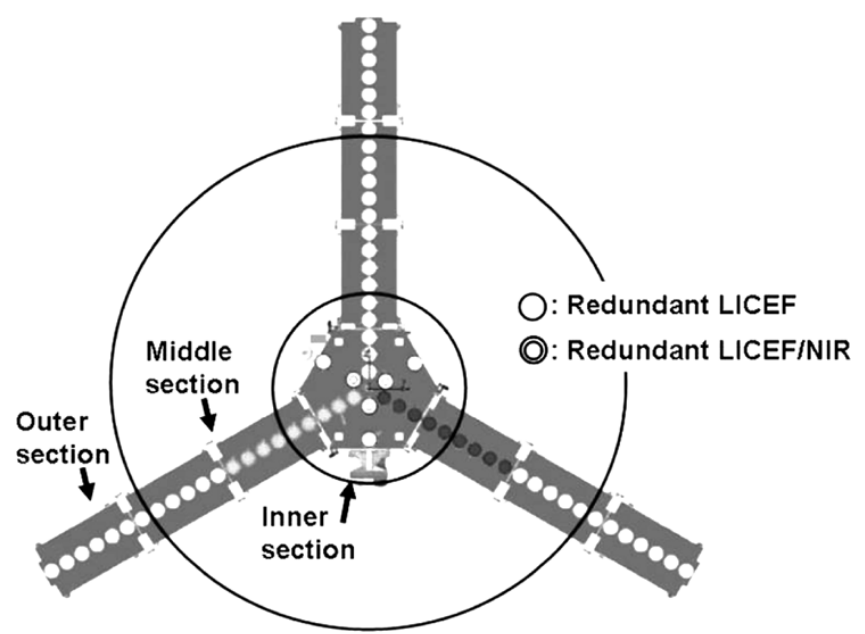

Fig. 1. SMOS Y-shaped array composed by $3 \times 23$ elements ( 15 in the hub, and six in each section of the arms). LICEF (LIght and Cost Effective Front-end) and LICEF/NIR (LICEF operating in Noise Injection Radiometer mode) shown in the figure. Circles limit the outer, middle, and inner section. Outer section includes elements numbers 14 to 21 in each arm. The middle section includes the elements from number 6 to 14, and the inner section contains the rest of the elements. Courtesy of EADS-CASA Espacio, Spain.

$\left(x_{1}, y_{1}\right)$ and $\left(x_{2}, y_{2}\right)$ are the phase centers position of each antenna, and $(\xi, \eta)=(\sin \theta \cos \phi, \sin \theta \sin \phi)$ are the direction cosines with respect to the $X$ and $Y$ axes.

\section{REQUiREMENTS ON THE MEASUREMENT OF THE ANTENNA VOLTAGE PATTERNS}

In the ideal case of identical antenna patterns and negligible fringe-washing effects $(\tilde{r} \approx 1)(1)$ is a Fourier transform. However, in the real case, antenna voltage patterns are different and must be accounted for in the image reconstruction process [2]. In [3], the radiometric accuracy degradation due to the imperfect knowledge of the antenna voltage patterns was derived. The sensitivity of the radiometric accuracy to the rms phase error is $1.11 \mathrm{~K} /{ }^{\circ}$, and that to the root mean square (rms) amplitude error $0.8 \mathrm{~K} / \%$ (linear units) [4]. The current measurement requirements for all the elements in the SMOS mission are $0.33^{\circ}$ for the rms phase error, and $0.05 \mathrm{~dB}(0.57 \%)$ for the rms amplitude error [4], in accordance with the conclusions of the SMOS Antenna Experts Meeting [5] for the ultimate achievable error in a near-field compact range.

\section{RADIOMETRIC ACCURACY DegRADATION DUE TO ANTENNA PATTERN ERRORS IN THE OUTER ELEMENTS}

From a low Earth orbit, the Earth occupies a large part of the field of view with smooth brightness temperature variations, and 

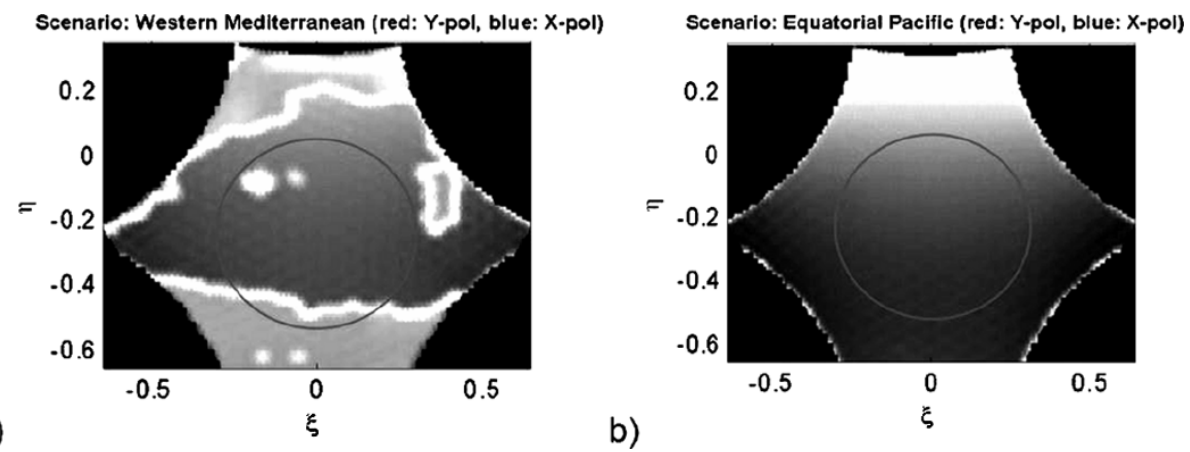

Fig. 2. SMOS alias-free FOV. (a) High-contrast brightness temperature scenario (western Mediterranean). (b) Low-contrast brightness temperature scenerio (equatorial Pacific). Y-polarization: antenna aligned with $\eta$ direction.
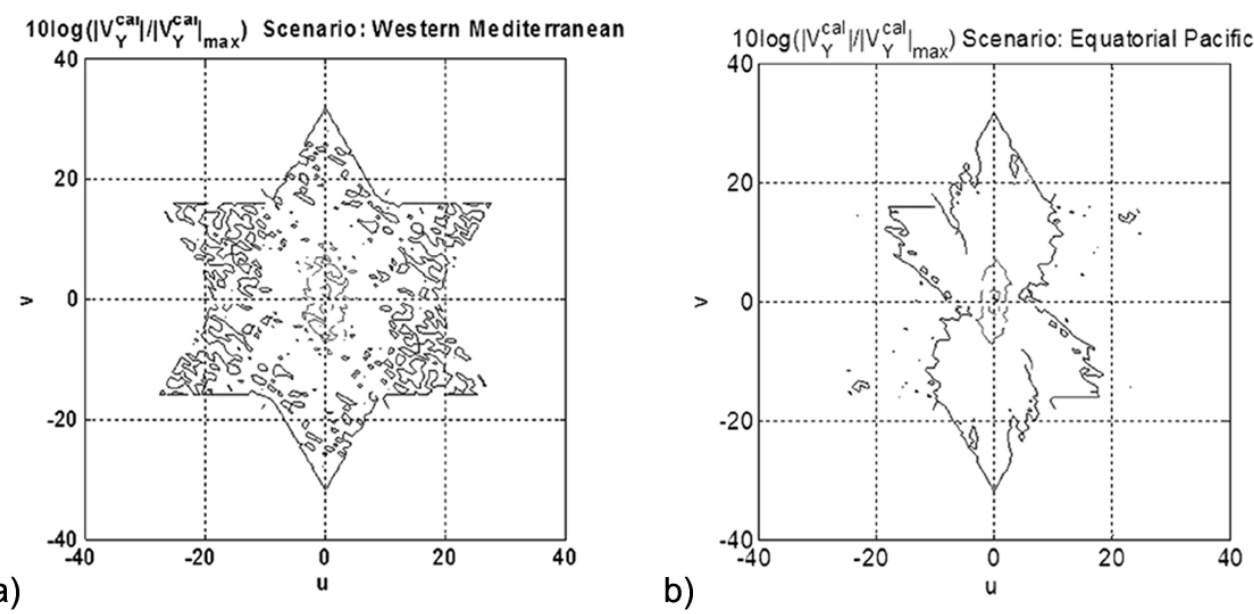

Fig. 3. Contour plots at $-3,-10$, and $-20 \mathrm{~dB}$ of the normalized visibility function corresponding to (a) Fig. 2(a) (antenna temperature equals $153.25 \mathrm{~K}$ ) and (b) Fig. 2(b) (antenna temperature equals $98.96 \mathrm{~K}$ ).

therefore, the visibility function exhibits a low-pass behavior, which depends on the type of scene being imaged. Since the antenna spacing in SMOS is $0.875 \lambda$, larger than $\lambda / \sqrt{3}$, there is aliasing in the imaging process [6], which limits the two-dimensional field of view (FOV) of the instrument. Fig. 2(a) and (b) shows two scenes only in the alias-free FOV and at Y-polarization: ${ }^{1}$ (a) a high-contrast scene with sharp brightness temperature transitions in the coast lines, while (b) shows a very homogeneous scene of the ocean. Fig. 3(a) and (b) shows the contour plots at -3 , -10 , and $-20 \mathrm{~dB}$ of the visibility function as would be measured by the SMOS/MIRAS array, normalized to their corresponding maxima. The star shape is clearly seen in Fig. 3(a), while it can barely be seen in Fig. 3(b). This indicates a truncation of the visibility function due to the limited size of the array: the star shape structure is clearly seen for a high-contrast scene with stronger high spatial frequency harmonics, and not for the low-contrast scene. ${ }^{2}$ This suggests that the antennas farther away from the hub may have a larger measurement error than the ones near the center, yet with a small impact in the radiometric accuracy.

In order to quantify this, a series of numerical simulations have been performed with the SMOS End-to-end Performance Simulator (SEPS) [8] taking into account exclusively antenna

\footnotetext{
${ }^{1}$ Y-polarization brightness temperature is a combination of vertical and horizontal polarizations over the Earth [7].

${ }^{2}$ Scenes with a higher contrast only happen naturally when the Sun is imaged. Even in this case, for $T_{\mathrm{SUN}}=200.000-2.000 .000 \mathrm{~K}$, the Sun's contribution is a constant visibility of $0.5-5 \mathrm{~K}$, on the order of the truncation error in Fig. 3(a) and (b).
}

pattern errors. The antenna voltage patterns used are the first nine which have been measured in [9]. They are all very similar, and in the boresight direction they exhibit a rms phase error of approximately $1.9^{\circ}$ to $3.1^{\circ}$, and a rms amplitude error of $0.65 \%$ to $0.89 \%$, depending on the polarization [4]. For the remaining antennas the antenna patterns have been repeated.

In the simulations, the computation of the visibility samples (1) has been performed with all the antenna patterns, but in the image reconstruction process the antenna voltage patterns of the $n$ outer elements of each arm have been replaced by the average antenna pattern in order to simulate antenna pattern errors. The radiometric accuracy degradation in one snapshot is computed as a function of $n$, as the rms difference between the reconstructed brightness temperature image and the reconstructed one when all the antenna patterns are known in the a circle centered at $(0,-0.237)$ with radius 0.3 , covering the central part of the alias-free FOV. ${ }^{3}$ Away from this region, errors are amplified by the inverse of the antenna radiation pattern times $\sqrt{1-\xi^{2}-\eta^{2}}$. Results at X-and Y-polarizations are shown in Fig. 4(a) and (b), corresponding to the scenes presented in Fig. 2(a) and (b) (only Y-polarization). As it can be noticed, for the high-contrast scene the radiometric accuracy degradation ${ }^{4}$ in the circular region

${ }^{3}$ Region selected by ESA to compute the SMOS error budget [4], since it contains approximately 3000 points (more than enough for statistical purposes), error amplification is moderate $\sim 1.5$, and it is far from the aliases tails.

${ }^{4}$ Same criterion for radiometric accuracy degradation as specified by ESA in [11]. 

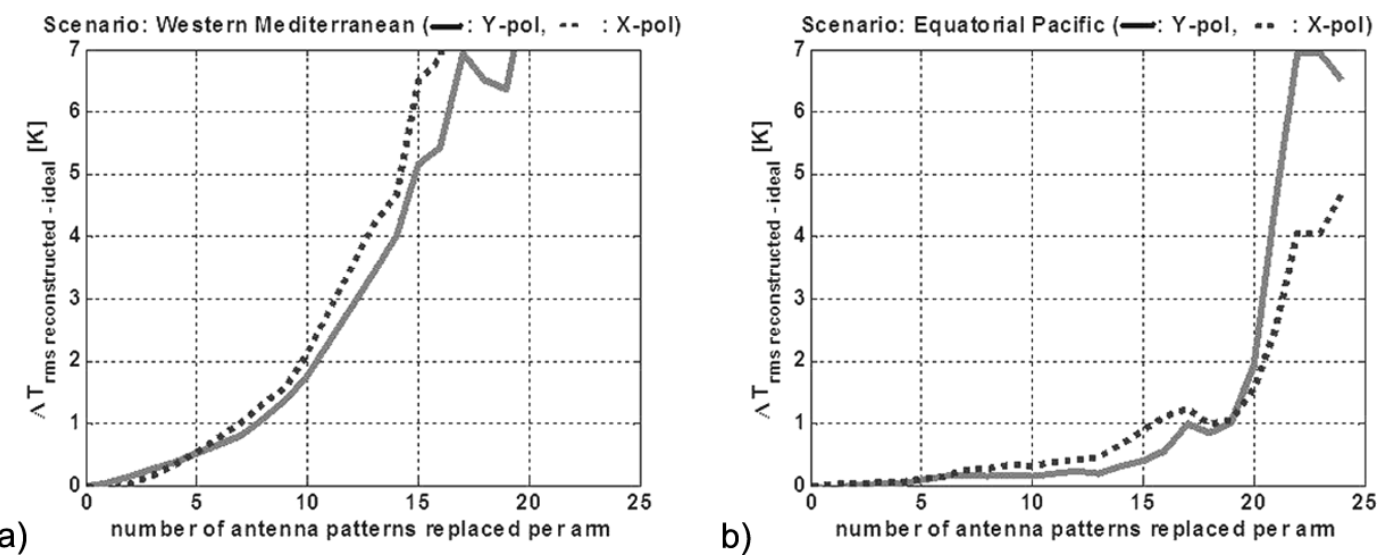

Fig. 4. Radiometric accuracy degradation in one snapshot. (Solid line) Y-polarization. (Dotted line) X-polarization) when the antenna voltage patterns of $n$ elements of each arm starting from the edge (abscissas axis) are replaced by the average antenna voltage pattern corresponding to (a) Fig. 2(a) and (b) Fig. 2(b).

marked in Fig. 2 is smaller than $1 \mathrm{~K}$ if the antenna patterns of the seven outer elements of each arm are replaced by the average pattern, that is their antenna pattern errors can be neglected. For the low-contrast scene the situation is even more favorable, since the antenna voltage pattern of the 16 outer elements can be replaced by the average pattern. Therefore, the antenna voltage pattern characterization efforts can be focused on the 48 inner elements of the array, without a significant contribution to the rms radiometric accuracy ( $\leq 1 \mathrm{~K}$ for high-contrast scenes). Note that in the most updated SMOS error budget [4] the snapshot radiometric sensitivity is $2.42 \mathrm{~K}$ and the rms radiometric accuracy is $2.87 \mathrm{~K}$. Therefore, a $1-\mathrm{K}$ contribution will lead to a total radiometric accuracy of $\sqrt{(2.87 \mathrm{~K})^{2}+(1 \mathrm{~K})^{2}}=3.04 \mathrm{~K}$, which means a degradation of just $0.17 \mathrm{~K}$.

\section{CONCLUSION}

Even though all the antenna patterns need to be measured to be sure that they behave properly, efforts can be focused in the measurement of the 48 inner antennas. Since the antenna coupling is very low, $\leq-32 \mathrm{~dB}$ for neighbor antennas $(d=0.88 \lambda)$, and $\leq-40 \mathrm{~dB}$ for antennas spaced $2 d=1.76 \lambda$ [9], the antenna voltage pattern of an element is modified by at most by the presence of the neighboring three antennas on either side [11], the antenna voltage pattern measurements can be performed for the 33 antennas in the hub and in the inner arm sections simultaneously, provided three dummy antennas of the middle section are mounted. Afterward, each of the three arms must be mounted individually to measure the middle sections, including the effect of the neighbor elements of the inner and outer sections. In this position, the outer sections can be also measured to check that they behave properly, but there is not strict limit in the required accuracy, since even if their voltage patterns were replaced by the average antenna voltage pattern of the inner sections, the ra- diometric accuracy will not be degraded more than $1 \mathrm{~K}$, even for high-contrast scenes.

\section{REFERENCES}

[1] I. Corbella, N. Duffo, M. Vall-llossera, A. Camps, and F. Torres, "The visibility function in interferometric aperture synthesis radiometry," IEEE Trans. Geosci. Remote Sens., vol. 42, no. 8, pp. 1677-1682, Aug. 2004.

[2] A. Camps, J. Bará, F. Torres, and I. Corbella, "Extension of the CLEAN technique to the microwave imaging of continuous thermal sources by means of aperture synthesis," Progr. Electromagn. Res., vol. PIER 18, pp. 67-83, Jan. 1998.

[3] A. Camps, J. Bará, F. Torres, I. Corbella, and J. Romeu, "Impact of antenna errors on the radiometric accuracy of large aperture synthesis radiometers. Study applied to MIRAS," Radio Sci., vol. 32, no. 2, pp. 657-668, Mar.-Apr. 1997.

[4] A. Camps, I. Corbella, F. Torres, M. Vall-llossera, and N. Duffo, SMOS system performance model and error budget Univ. Politècnica Catalunya, Barcelona, Spain, SO-TN-UPC-PLM-02 rev 3.20, Nov. 6 , 2003.

[5] N. Skou, "See papers," in the SMOS Ground Calibration Plan Minutes of the Antenna Expert Meeting, Tech. Univ. Denmark, Lyngby, May 14, 2002, unpublished.

[6] A. Camps, I. Corbella, M. Vall-1lossera, N. Duffo, F. Marcos, F. Martínez-Fadrique, and M. Greiner, "The SMOS end-to-end performance simulator: Description and scientific applications," in Proc. IGARSS, Toulouse, France, Jul. 21-25, 2003, vol. 1, pp. 13-15.

[7] M. Martín-Neira, S. Ribó, and A. J. Martín-Polegre, "Polarimetric mode of MIRAS," IEEE Trans. Geosci. Remote Sens., vol. 40, no. 8, pp. 1755-1768, Aug. 2002.

[8] A. Camps, J. Bará, I. Corbella, and F. Torres, "The processing of hexagonally sampled signals with standard rectangular techniques: Application to 2-D large aperture synthesis interferometric radiometers," IEEE Trans. Geosci. Remote Sens., vol. 35, no. 1, pp. 183-190, Jan. 1997.

[9] EADS-CASA, MIRAS image validation: Measured antenna data (CDROM), Jul. 29, 2002.

[10] M. Vall-llossera, N. Duffo, A. Camps, I. Corbella, F. Torres, and J. Bará, "Reliability analysis in aperture synthesis interferometric radiometers: Aplication to L-band MIRAS instrument," Radio Sci., vol. 36, no. 1, pp. 107-117, January 2001.

[11] A. Camps, F. Torres, I. Corbella, J. Bará, and P. de Paco, "Mutua coupling effects on antenna radiation pattern: an experimental study applied to interferometric radiometers," Radio Sci., vol. 33, no. 6, pp. 1543-1552, Nov.Dec. 1998. 